\title{
The central dialysis fluid delivery system (CDDS): is it specialty in Japan?
}

\author{
Hideki Kawanishi ${ }^{1} 2^{*}$ (D, Misaki Moriishi ${ }^{3}$, Naoko Takahashi ${ }^{4}$ and Shinichiro Tsuchiya ${ }^{1}$
}

\begin{abstract}
The central dialysis fluid delivery system (CDDS) simplifies the maintenance and supervision involved by enabling the combined management of dialysis fluid for multiple persons, preparation of cleaning and antiseptic solutions, and delivery of these to each patient monitor. The CDDS has been used exclusively in Japan since 1960s. Approximately $88 \%$ of dialysis machines are patient monitors with CDDS. It is a cost-effective, laborsaving, time-tested system with good microbial safety, which has been used for 45 years. In many countries, especially in Asia, the number of end-stage renal disease (ESRD) patients is increasing. The CDDS will contribute to such emerging situations with its easy handling and economic advantages.
\end{abstract}

Keywords: Hemodialysis, Dialysis fluid delivery system, Central dialysis fluid delivery system (CDDS), Dialysis fluid purification

\section{Background}

It is widely known that the survival rate of Japanese hemodialysis patients is the highest in the world [1]. This is mainly due to the development of dialysis and blood purification devices and development of the dialysis system. Cooperation among academic societies, clinical engineering technologists, and industries has also played a significant role. The introduction of the central dialysis fluid delivery system (CDDS) in the 1960s enabled the provision of stable dialysis conditions for all patients, which made a marked contribution to the field of dialysis.

In comparison to single-patient dialysis fluid delivery system (SPDDS), the central dialysis fluid delivery system (CDDS) simplifies the maintenance and supervision involved by enabling the combined management of dialysis fluid for multiple persons, preparation of cleaning and antiseptic solutions, and delivery of these to each patient monitor. Reliability is required in CDDS systems, as a single abnormality can affect multiple patients negatively. In Japan, CDDS has evolved from more than 45 years of experience. In many countries, especially in Asia, the number of end-stage renal disease (ESRD) patients is increasing [2]. CDDS will contribute to such

\footnotetext{
* Correspondence: h-kawanishi@tsuchiya-hp.jp

${ }^{1}$ Tsuchiya General Hospital, 3-30 Nakajima-cho, Naka-ku, Hiroshima 730-8655, Japan

${ }^{2}$ Faculty of Medicine, Hiroshima University, Hiroshima, Japan

Full list of author information is available at the end of the article
}

emerging situations with its easy handling and economic advantages.

\section{History of clinical hemodialysis and development of CDDS}

Willem Kolff developed the first successful dialysis treatment. Dr. Kolff used a rotating drum kidney to treat a 67-year-old patient who had been admitted to the hospital with acute kidney failure in 1945 [3]. The success was partially due to the technical improvements in the actual equipment used for the treatment. Kolff's rotating drum kidney used membranous tubes made from a new material known as cellophane. As the membranous tubes passed through the bath, the uremic toxins would pass into this rinsing liquid using the abovementioned physical principles. The Kolff-Brigham kidney passed its practical test under extreme conditions during the Korean War. Since then, it has been widely used worldwide as the SPDDS [4].

The developments that began with the Quinton-Scribner Shunt allowed the long-term treatment of patients with chronic kidney failure. In the spring of $1960[5,6]$, Belding Scribner implanted a shunt in the American, Clyde Shields, in Seattle. Mr. Shields became the first chronic hemodialysis patient, and the dialysis treatments allowed him to live an additional 11 years before dying of cardiac disease in 1971. Dr. Scribner developed the dialysis 
system, including the CDDS [7]. Our Tsuchiya dialysis center was eventually opened and has been operational since 1967. The Sweden Seattle-CDDS, Sweden Freezerpatient monitor, and the Kiil dialyzer developed by Dr. Scribner are used in this dialysis center [8]. This was the first dialysis center in Japan where the CDDS was used, and hemodialysis was performed $24 \mathrm{~h}$ a day, 6 days a week.

Data from the Japanese society for dialysis therapy (JSDT) showed that there were only 110 patient monitors in 50 facilities, and the CDDS was used in $56 \%$ of the cases in Japan in 1968. By the end of 2014, the number of patient monitors and dialysis facilities had increased to 130,952 in 4314 facilities, respectively, with $90 \%$ of patients undergoing treatment using the CDDS [9].

\section{Dialysis fluid delivery systems}

Currently available dialysis fluid delivery systems include the single-patient dialysis fluid delivery system (SPDDS) (or individual dialysis fluid delivery system) and central concentrates delivery systems (CCDS), as well as the CDDS (Fig. 1) [10, 11].

In SPDDS, the patient monitor contains a dialysis fluid supply equipment. Presently, it is widely used in countries other than Japan and is considered as the global standard for dialysis treatment. Although many models require separate water treatment equipment, a model that has this built in is also available. Aside from the advantage of the relatively free location, SPDDS allows for the individualization of dialysis fluid composition in order to meet unique patient needs.

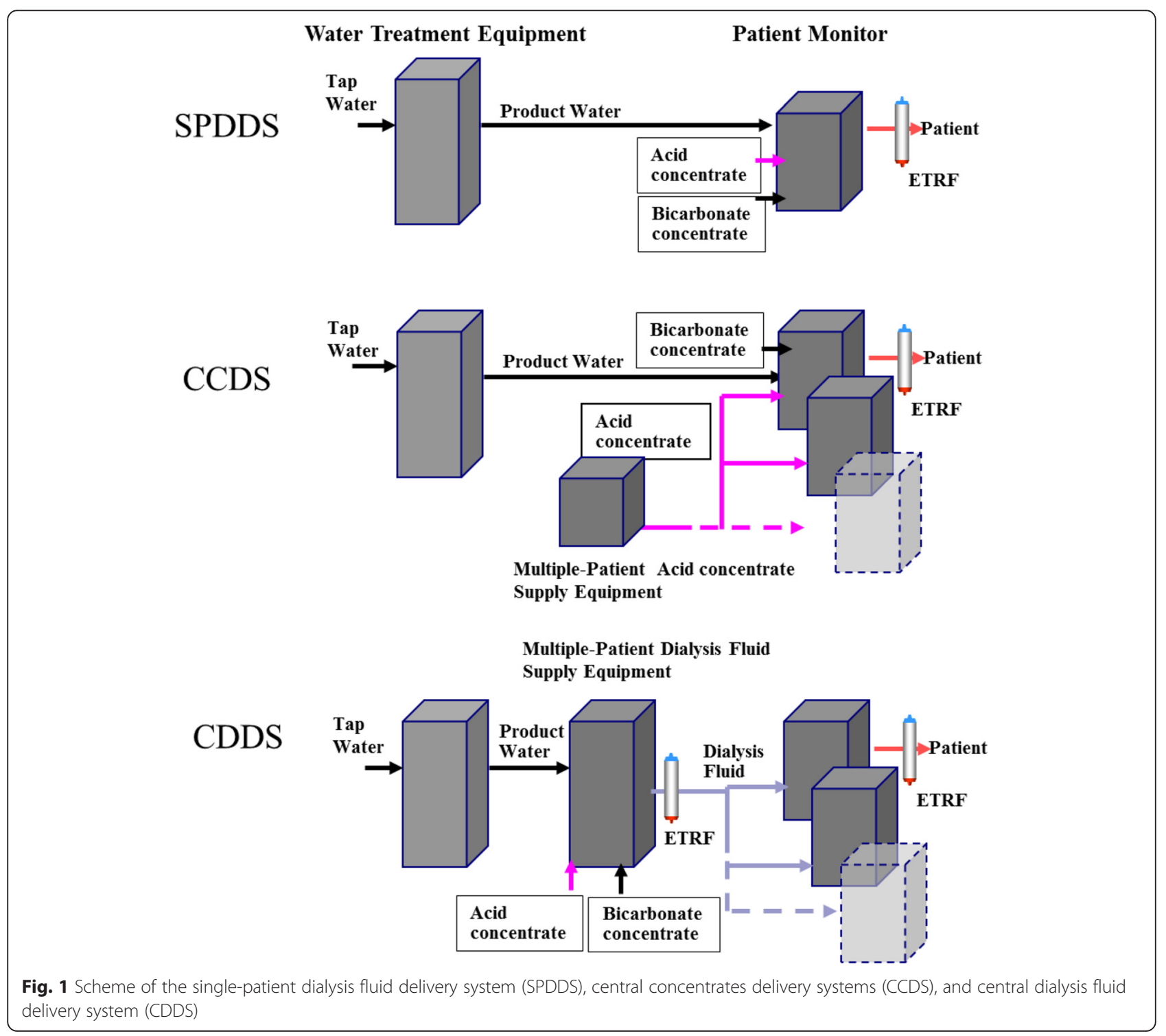


The CCDS supplies the acid concentrate (solution A) by multiple-patient acid concentrate supply equipment, and the bicarbonate concentrate (solution B) is intended to provide individual patient monitor. Different electrolyte concentrations of solution A are piped in and changed according to the patient's condition. CCDS is easy to maintain since it purifies in the long pipe in order to contain the acid in solution A and, possibly, in storage. The fact that CCDS can be used in the temporary treatment of many patients remains a distinct advantage. It also allows the additional benefit of variation of the dialysis fluid compositions and has been used in the USA and countries such as China, where the number of patients is increasing.

The CDDS consists of the water treatment and multiplepatient dialysis fluid supply equipment (MPDFSE), as well as the patient monitor. Although the ability to supply a large amount of dialysis fluid is only temporary in CDDS, it is possible to use many patient monitors [12, 13].

One advantage of the CDDS is that it is usually more cost effective (Table 1) [10]. Maintenance is simple and easy and not labor-intensive for the medical staff, if the system is placed completely. However, one disadvantage is that malfunction of the central proportioning unit will simultaneously affect several patients resulting in the discontinuation of dialysis at the patient station. Also, central systems do not allow for individualization of the dialysis fluid compositions to meet the patients' unique needs. Additionally, machine dysfunction affects all patients and there is the constant risk of contamination with the use of the long dialysis piping. Given these disadvantages, CDDS is no longer used in countries other than Japan.

\section{Why is CDDS used in Japan?}

Despite its disadvantages, CDDS has been widely accepted in Japan primarily because of the need for minimal space and cost saving options, in order to address the rapid increase in the number of dialysis patients. Unlike other countries, Japan has many the dialysis device-related

Table 1 Advantages and disadvantages of CDDS compared single-patients system

Advantages
- Cost effective
- Maintenance is simple and easy, if the system is placed completely
- Labor saving of medical staff
- Possibility of automated operation system
Disadvantages
- Dialysate composition; no permission to individual
- Machine dysfunction affects all patients
- Microbial: risk of long dialysis piping for contaminations

manufacturers with technical capabilities. Moreover, the clinical engineering technology system in Japan has developed specialist maintenance management expertise of dialysis devices.

\section{Dialysis fluid purification}

One problem that is unique to Japan is the process by which the CDDS should be guaranteed by manufacturers. Many parts of the CDDS are not approved as medical devices. Since they are manufactured by different companies, it is difficult to identify the company that is actually responsible for the device. Although the endotoxin retentive filter (ETRF), which is important for water and dialysis fluid purification control, has been determined as an industrial standard, there is no currently available standard for its management and application. This must be determined according to the degree of purity upstream and treatment mode.

In order to solve these issues, the JSDT published the "Standard of microbiological management of fluid for hemodialysis and related therapies" in 2007 (Table 2) [14] and the "Standard on the management of endotoxin

Table 2 Microbiological Quality Standard for Dialysis Fluids in Japan, excerpt from reference [14]

Attainment level

Dialysis water (reverse osmosis (RO) water)

Bacteria $<100 \mathrm{CFU} / \mathrm{ml}$

Endotoxin $<0.050 \mathrm{EU} / \mathrm{ml}$

Standard dialysis fluid

Bacteria $<100 \mathrm{CFU} / \mathrm{ml}$

Endotoxin $<0.050 \mathrm{EU} / \mathrm{ml}$

Ultrapure dialysis fluid

Bacteria $<0.1 \mathrm{CFU} / \mathrm{ml}$

Endotoxin $<0.001 \mathrm{EU} / \mathrm{ml}$ (less than detection limit)

Online-prepared substitution fluid

Sterile and non-pyrogenic

Bacteria $<10^{-6}$

Endotoxin $<0.001 \mathrm{EU} / \mathrm{ml}$ (less than detection limit)

Indications for dialysis system based on the quality of dialysis fluids

Standard dialysis fluid

Minimum requirement for dialysis therapy

Ultrapure dialysis fluid ${ }^{a}$

Dialysis fluid for the preparation of online substitution fluid.

Dialysis system for active use of back filtrate

Push and pull HDF system

Internal filtration-enhanced dialysis (IFEHD)

Online-prepared substitution fluid

Online HDF/online hemofiltration

aUltrapure dialysis fluid is desirable for all dialysis modalities 
retentive filter for dialysis and related therapies" in 2011 (Table 3) [15]. These were developed in accordance with the International Organization for Standardization (ISO) [16] and elaborates on well management in the CDDS by incorporating the concept of "validation."

Validation is a concept that was developed for the assurance of system compatibility and product quality and includes the validation of the manufacturing and quality control methods (i.e., the system design and equipment of the manufacturing facility, manufacturing procedure and processes). Confirmed results must yield acceptable limits, and this must be documented in writing [17]. CDDS are used in many dialysis facilities, where the dialysis fluids are prepared through multiple processes performed by various apparatuses connected in series. These apparatuses are selected and arranged by each dialysis facility. In such a manufacturing system, a validation concept is necessary for process management and product quality assurance. A dialysis facility functions as a

Table 3 Standard on the management of endotoxin retentive filter (ETRF), excerpt from reference [15]

Definition of ETRFs and required performance

- Endotoxin retentive filters generally refer to the filters used to remove ET and microorganisms from dialysis fluid for their purification. This ETRF Management Standard addresses the management of an ETRF installed immediately before the dialyzer in a bedside dialysis console.

- An ETRF should maintain LRVs of at least 2 for ET and at least 4 for bacteria to obtain ultrapure dialysis fluid, assuming that the dialysis fluid before the ETRF maintains its microbiological quality at values for standard dialysis fluid specified in the JSDT Standard.

Standard for handling ETRFs

- Manufacturers' instructions for the ETRF installation shall be observed.

- To obtain ultrapure dialysis fluid sample, the dialysis fluid at the inlet of the ETRF should be maintained within the allowable level for standard dialysis fluid specified in the JSDT Standard.

Cleaning and disinfection of ETRFs

- Dialysis machines shall be cleaned and disinfected following the methods recommended by the manufacturer.

- The inside and outside of hollow fibers shall be cleaned and disinfected during the cleaning and disinfection of ETRFs.

-When cleaning and disinfection methods other than those recommended by the manufacturer are used, the durability of ETRFs shall be tested at each facility to confirm their safety.

\section{Duration of ETRF use}

- The duration of ETRF use recommended by the manufacturer shall be observed when the dialysis fluid flowing into the ETRF cannot be maintained within the allowable levels for standard dialysis fluids as specified in the JSDT Microbiological Quality Standard for Dialysis Fluids.

- The duration of ETRF use should be determined by the Dialysis Equipment Safety Management Committee when the standard dialysis fluid falls within allowable levels.

Monitoring and durability test of ETRFs

- When ETRFs are used under conditions other than those recommended by the manufacturer, their durability shall be tested at each facility to confirm their safety. dialysis fluid manufacturing facility and is responsible for the final quality of the dialysis fluid. Therefore, the person who is responsible for manufacturing the device, including the managers of the various processes, must be appointed for the dialysis fluid purification process in the same manner as those performed in manufacturing facilities. At a dialysis facility where dialysis fluid is also manufactured, the following requirements of Table 4 must be established as validation processes for the dialysis fluid purification procedure. If the results of comprehensive evaluation of these processes meet the requirements, the dialysis fluid preparation system is considered to have been validated.

Previously, there were also the concerns about the need to emphasize complete purification from the upstream of the dialysis fluid supply system to the end of the downstream flow. The concept of validation assumes that, because contamination is present, it is important to construct a system that always remains unaffected by the contamination downstream, in order to isolate the degrees of contamination to each area. Figure 2 demonstrates the concept of sterility in each area on the CDDS [13]. The concept of preventing pollution by isolating the degree of contamination to each the individual terminal area is propagated.

\section{The standard on the management of ETRF}

Some basic terms and conditions, such as piping management, disinfection methods, and equipment management that were defined in order to achieve purification of the dialysis fluid, will eventually depend on the performance of the ETRF. Currently, newly developed dialysis equipment, such as the online hemodiafiltration (HDF) system, are secured by being approved as a medical device in a state where the ETRF is incorporated for purification. However, the performance of the ETRF that have been incorporated into other systems is defined by industry standards. Since it has not been recognized as a medical instrument, provisions for its use in dialysis equipment

Table 4 The requirement as validation processes for the dialysis fluid purification procedure

- Intended of quality of purification of dialysis fluid

- Validity of equipment, preparation processes, products, and plan of the preparation method (system design of apparatuses)

- Confirmation of the designs of the equipment, processes and preparation methods, and their completion as designed (installation qualification)

- Confirmation of the utility of the equipment, processes, and methods, in order to achieve the intended objective (operational qualification)

- Testing of the system under the conditions of actual preparation and confirmation of the operation of the system as designed (performance qualification) (periodic evaluation of the quality of supply water, RO water, and dialysis fluid)

- Guarantee the disinfection of the interior of the system during non-use periods 


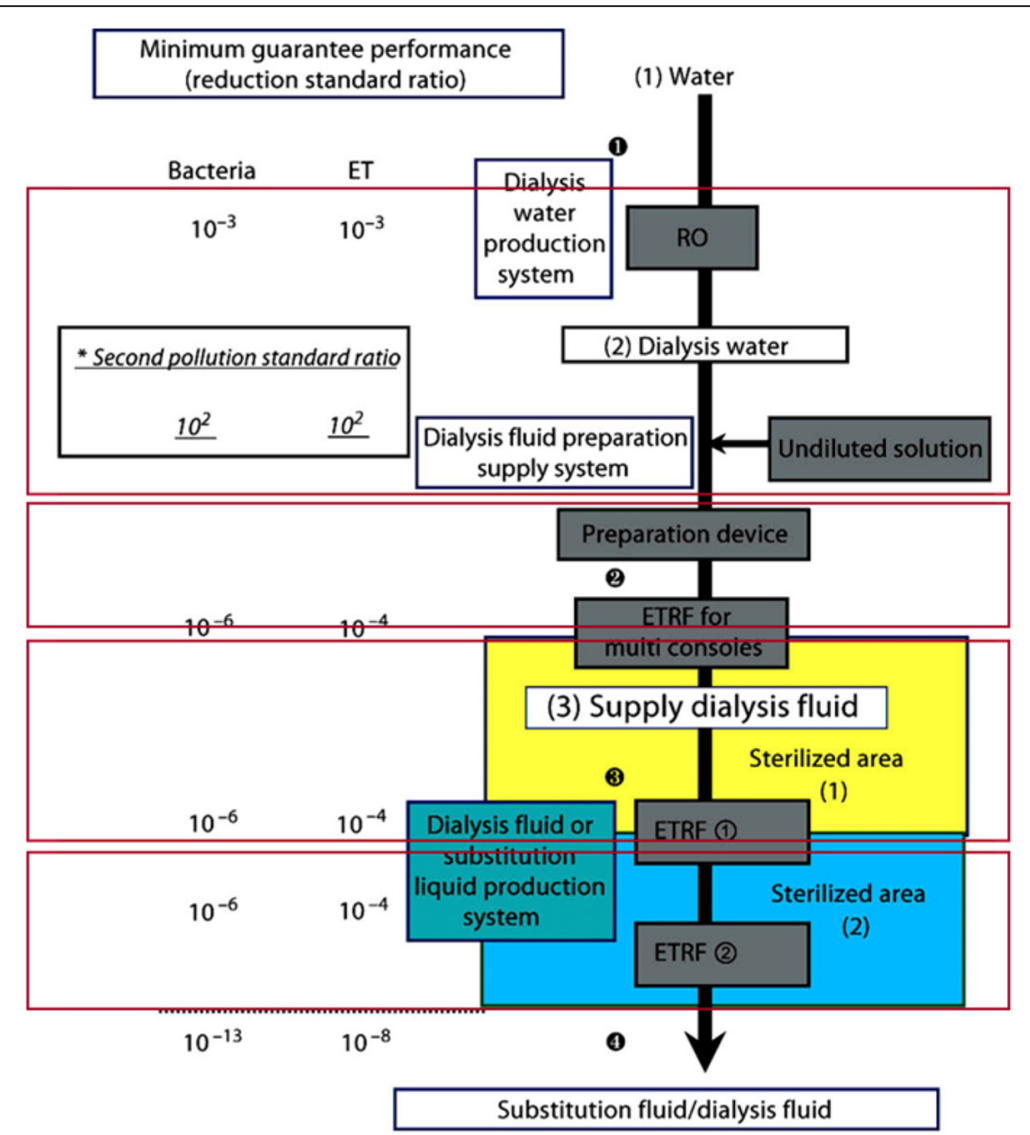

Fig. 2 Validation of management for microbial contamination in CDDS

have not been determined and are the responsibility of the individual dialysis facilities. In response, the JSDT published the "Standard on the management of endotoxin retentive filter for dialysis and related therapies." Table 3 shows the excerpt of the final document, which is applicable to its clinical use [15]. Dialysis fluid flowing into ETRF is assumed to be of the "standard quality" of dialysis fluid (bacteria $<100 \mathrm{CFU} / \mathrm{ml}$, endotoxin $<0.050 \mathrm{EU} / \mathrm{ml}$ ) that complies with the JSDT standards [14].

\section{Actual management of CDDS}

As described, water quality management is the responsibility of the dialysis equipment safety management committee of each facility. Therefore, the commission has drafted a management manual and organized a committee, which regularly scrutinizes for compliance.

\section{The setting of management target value}

CDDS is composed of different purification areas, extending from the upstream and to the downstream areas (Fig. 2). Basic management entails determination of the purification of an area, to ensure the maintenance of an acceptable level of purification. In order to determine this, the target quality standards in each area need to be identified. Reference values for each area do not always need to be set at the highest levels. According to the purification status of the facility, the value obtained for the highest level (e.g., ultrapure quality) of purity in the final dialysis fluid is determined.

\section{Determination of management and the confirmation methods}

Based of the state of purification of each area, the management (purification and disinfection methods, frequency of replacement of the ETRF) and verification (collection, measurement, and frequency of ET and microbial level determination) methods need to be determined.

\section{Measures of the region exceeds the reference value}

The purifying measures are necessary if the measured value of the areas reaches the action level of the reference value. The action level is set according to the quality condition of each facility and is typically set at $50 \%$ of the maximum allowable level, except for endotoxin levels in ultrapure dialysis fluid [14]. In this case, the purification and disinfection methods and quality of the reverse osmosis (RO) membrane and ETRF are 
reexamined and regularly monitored in order to secure the reference value.

\section{Judgment of the treating continuation propriety}

Even if each area is unable to secure the reference values, the final dialysis fluid that reached the reference level values needs to be identified, in order to continue treatment. However, if the final dialysis fluid is below the reference level, consideration should be given to changing the way dialysis is conducted (i.e., stopping the use of the super high-flux dialyzer by the dialysis equipment safety management committee).

Further installation, purification, and disinfection methods, with respect to its duration of use, were determined according to the manufacturer's recommendations. If each facility decides to change the purification/disinfection methods and the use periods, the dialysis equipment safety management committee in each facility were responsible for addressing these issues.

\section{Clinical engineering technologists system}

Factors that affect the quality of CDDS maintenance in Japan include clinical the engineering technology systems that maintain these dialysis system using key operations. This is a national qualification that combines both medicine and engineering based on the "clinical engineering technologists act," which came into effect in 1987. Among the 2300 clinical engineering technologists who obtain this qualification yearly, more than $80 \%$ are involved in blood purification field.

\section{Outline of the newly development of CDDS}

Figure 3 describes the processes used by the CDDS to achieve dialysis fluid purification in a flow chart [18-20]. The source water (main tap water is generally used) is taken up into the water treatment system and preprocessed through the water softener and activated carbon adsorption filter. This water is then purified into dialysis water using a RO module, which is almost completely free of leaks with a sodium chloride blocking rate of over $99.5 \%$.

The dialysis water then accumulates in the dialysis water tank. In order to prevent cross-contamination of the dialysis water, water is removed from each line at the startup of the RO equipment. Any remaining water is expelled when the equipment is stopped. Dialysis water is cycled to the RO module while the equipment is in operation, even when purification is not occurring, in order to prevent water from pooling inside the equipment. With regard to the dialysis water supply lines, an ETRF is placed at the re-entry mouth of the dialysis water tank as a looped pipe, in order to provide circulation to the dialysis water tank, countering the presence of contaminants inside the pipes and preventing crosscontamination.

The powdered acid concentrate (solution A) and bicarbonate concentrate (solution $\mathrm{B}$ ) are used to adjust the dialysis fluid. In the CDDS, a powder-mixing device was used to dissolve powder A (acid concentrate dry) and powder B (bicarbonate concentrate dry) in the dialysis water, which was then supplied with the dialysis water to

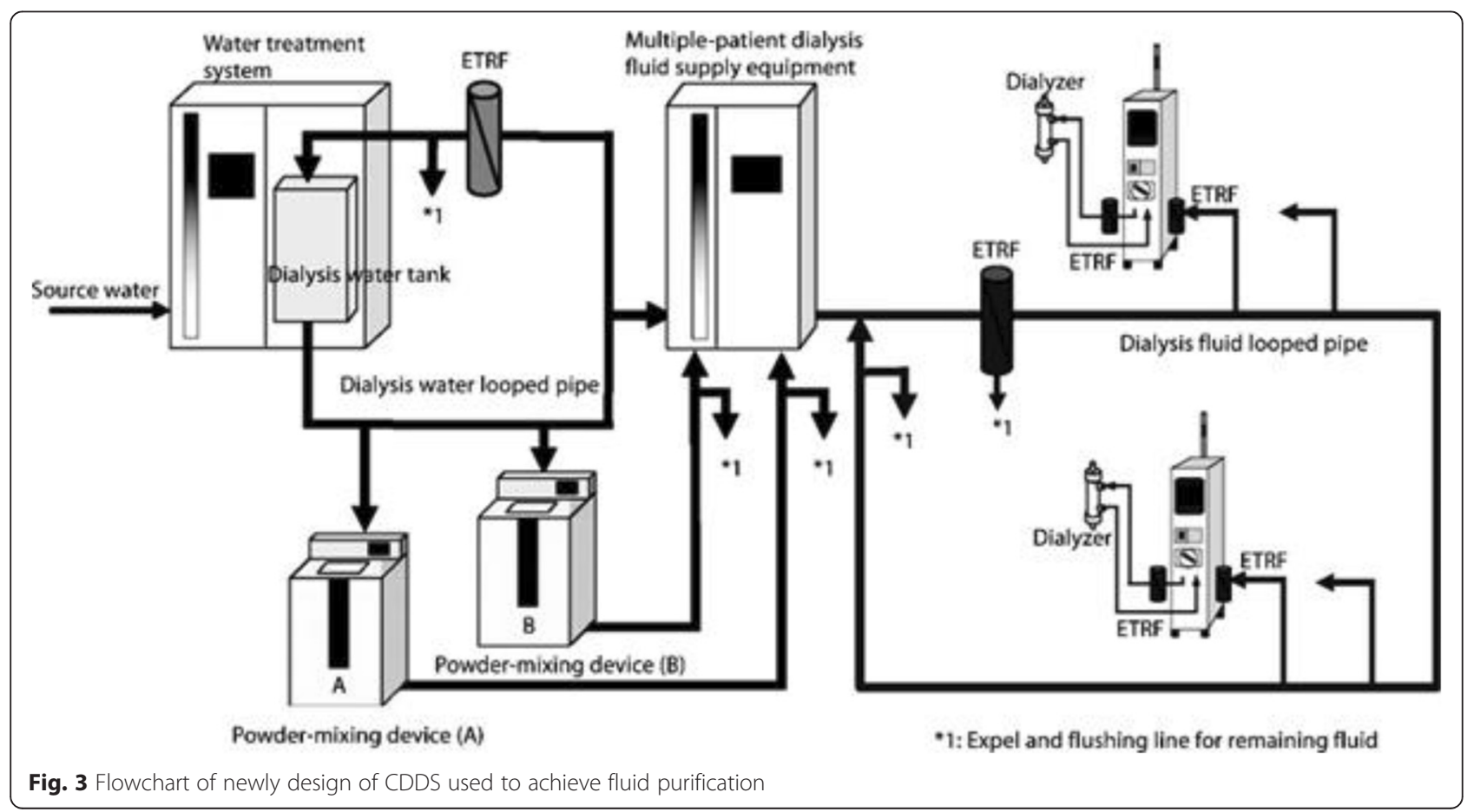


MPDFSE, mixed to a preset ratio, and supplied as dialysis fluid to each patient monitor. The pipe connecting the MPDFSE to each patient monitor also has a looped circulation pump in place. This prevents cross-contamination by eliminating dead-end pipes and causing dialysis fluid to circulate back, if the flow rate goes above a set level, preventing water accumulation. ETRF are also placed immediately after the dialysis fluid inlet of each patient monitor and looped dialysis fluid pipe, as well as immediately before the dialyzer. When each patient monitor is started, displacement washing occurs with the purified dialysis fluid.

\section{Basic disinfection and cleaning method}

After the hemodialysis treatment of the day is completed, all the patient monitors are cleaned, according to a standard procedure, using a sodium hypochlorite solution, which is adjusted to within 200-500 ppm by the MPDFSE. A weak concentration (50 ppm or less) of sodium hypochlorite solution is then substituted through the inside of the MPDFSE, the supply lines, and the patient monitors and maintained until the beginning of the cleaning process prior to the next round of hemodialysis [19].

A concentration of $1 \%$ acetic acid is also used to wash the MPDFSE twice a week, following the above process, in order to preventing calcium carbonate scaling inside the equipment and pipes due to the dialysis fluid. In such cases, the sterilization process first mentioned occurs after cleaning with acetic acid.

\section{Newly development of Fully automated dialysis system (FADS)}

The priming, guiding of blood into the dialyzer, and blood rinse back for hemodialysis treatment require a certain level of expertise which has proven to be a stumbling block to the development of automation. A system utilizing backfiltrated dialysis fluid or substitution fluid by online process as a means of standardizing and reducing the labor involved in these processes has thus been developed [18-20]. These systems makes active use of the backfiltrated dialysis fluid or substitution fluid by online process and requires strict control of water quality for each patient monitor and the CDDS, as well as purification of the dialysis fluid.

The FADS was able to actively use purified dialysis fluid through backfiltration or substitution fluid to automate the priming, blood rinse back, and rapid fluid replenishment processes, with each process segueing to the next through the touch of a single button (Fig. 4).

The advantages of the FADS in clinical terms are the shortened time required for start-up and blood rinse back and the simplification of action needed for rapid fluid replenishment, rendering hemodialysis operable by staff, regardless of their level of experience. This equipment also allows the separation of duties for needle insertion/removal and startup/blood rinse back, so that dialysis staff with long experience can handle the needle insertion/removal while staff with less experience can handle startup/blood rinse back. This can reduce the number of staff needed for each dialysis session and saves on labor costs $[18,19]$. The hermetically sealed nature of the blood circuit, from needle insertion to the end of hemodialysis treatment, has also reduced the risk of incidents involving air bubbles or blood contamination. Human errors are minimized through the simplification of the processes involved in hemodialysis treatment. The number of accidents reported that have occurred due to less experienced staff has decreased since the introduction of the FADS.

\section{Conclusions}

CDDS has been used exclusively in Japan since 1960s. Approximately $88 \%$ of dialysis machines are patient monitors with CDDS. It is a cost-effective, laborsaving, time-tested system with a good microbial safety profile,

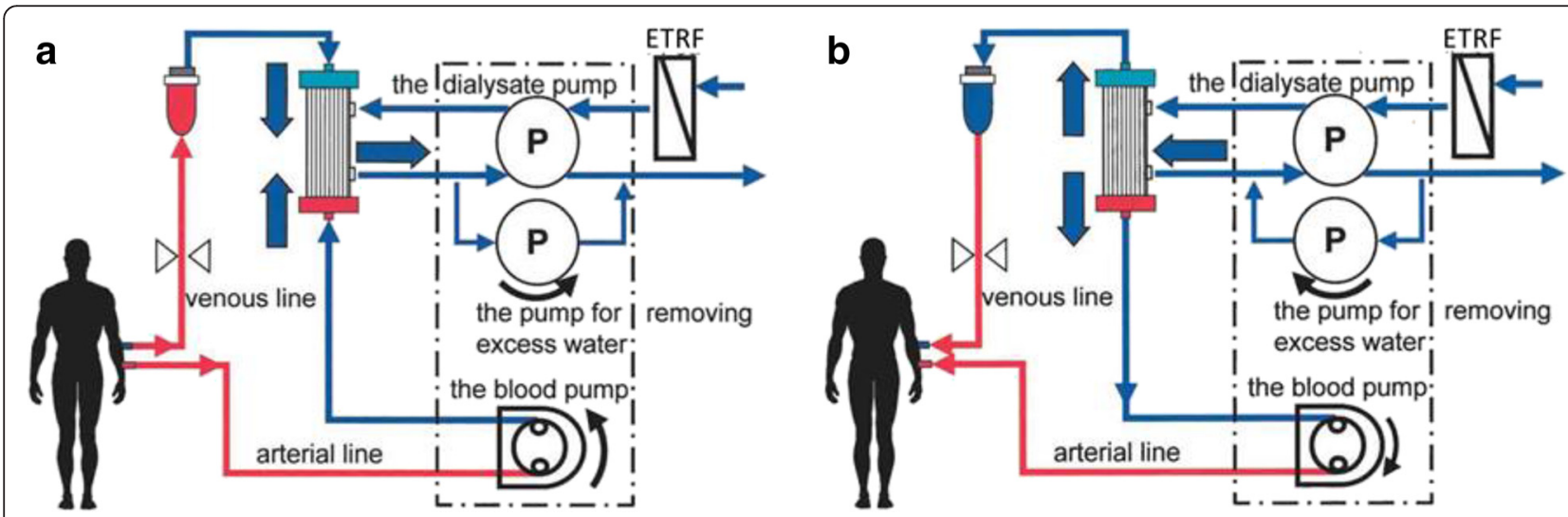

Fig. 4 Fully automated dialysis system (FADS) a Automated guiding of blood into the dialyzer. Red lines indicate the flow of blood. Blue lines indicate the flow of the dialysate. Thick blue arrows indicate the direction of dialysate flow. $\mathbf{b}$ Blood return and collection. Red lines indicate the flow of blood, and blue lines indicate the flow of the dialysate. Thick blue arrows indicate the direction of the dialysate flow 
which has been defined from 45 years of experience. In many countries, especially in Asia, the number of ESRD patients is increasing [2]. The CDDS will contribute to such emerging situations with its easy handling and economic advantages.

\section{Competing interests}

The authors declare that they have no competing interests.

\section{Authors' contributions}

HK carried out this study and drafted the manuscript. MM, NT, and ST have to manage the actual system. All authors read and approved the final manuscript.

\section{Author details}

'Tsuchiya General Hospital, 3-30 Nakajima-cho, Naka-ku, Hiroshima 730-8655, Japan. ${ }^{2}$ Faculty of Medicine, Hiroshima University, Hiroshima, Japan. ${ }^{3}$ Nakajima Tsuchiya Clinic, Hiroshima, Japan. ${ }^{4}$ Omachi Tsuchiya Clinic, Hiroshima, Japan.

Received: 15 September 2015 Accepted: 5 October 2015

Published online: 28 January 2016

\section{References}

1. Goodkin DA, Bragg-Gresham JL, Koenig KG, Wolfe RA, Akiba T, Andreucci VE, et al. Association of comorbid conditions and mortality in hemodialysis patients in Europe, Japan, and the United States: the dialysis outcomes and practice patterns study (DOPPS). J Am Soc Nephrol. 2003;14:3270-7.

2. Fresenius Medical Care. ESRD patients in 2013, http://www.vision-fmc.com/ files/ESRD_Patients_in_2013.pdf

3. Kolff W. New ways of treating uremia. London: J.A Churchill; 1946.

4. Merrill JP, Thorn GW, Walter CW, Callahan EJ, Hollingsworth Smith Jr L. The use of an artificial kidney. J Clin Invest. 1950;29:412-24.

5. Quinton W, Dillard D, Scribner BH. Cannulation of blood vessels for prolonged hemodialysis. Trans Am Soc Artif Intern Organs. 1960;6:104-13.

6. Scribner BH, Caner JE, Buri R, Quinton W. The technique of continuous hemodialysis. Trans Am Soc Artif Intern Organs. 1960;6:88-103.

7. Grimsrud L, Cole JJ, Lehman GA, Babb AL, Scribner BH. A central system for the continuous preparation and distribution of hemodialysis fluid. Trans Am Soc Artif Intern Organs. 1964;10:107-9.

8. Kawanishi H. History and Development of Tsuchiya General Hospital in Hiroshima. Blood Purif. 2015; 40: DOI: 10.1159/000439243

9. The Japanese Society for Dialysis Therapy, editor, illustrated overview of regular dialysis treatment in Japan, as of 31 December 2014. http://docs.jsdt. or.jp/overview/index.html (in Japanese).

10. Keshaviah P. Technology aspects of hemodialysis and peritoneal dialysis. In: Nissenson AR, Fine RN, Gentle DE, editors. Clinical dialysis. 3rd ed. USA: Prestice-Hall International Inc; 1995. p. p46-76.

11. Friedman EA, Lundin AP. Dialysate delivery: historical, theoretical, and practical aspects. Kidney Int Suppl. 1980;10:S33-43.

12. Koda $Y$, Mineshima M. Advances and advantages in recent central dialysis fluid delivery system. Blood Purif. 2009:27 Suppl 1:23-7.

13. Tomo T, Shinoda T. Standardization of water purification in the centra dialysis fluid delivery system: validation and parametric method. Blood Purif. 2009:27 Suppl 1:36-40

14. Kawanishi H, Akiba T, Masakane I, Tomo T, Mineshima M, Kawasaki T, et al. Standard on microbiological management of fluids for hemodialysis and related therapies by the Japanese Society for Dialysis Therapy 2008. Ther Apher Dial. 2009:13:161-6.

15. Masakane I, Kawanishi H, Mineshima M, Takemoto Y, Uchino J, Hoshino T, et al. 2011 JSDT standard on the management of endotoxin retentive filter for dialysis and related therapies. Ther Apher Dial. 2013;17:229-40.

16. ISO 23500:2014 Guidance for the preparation and quality management of fluids for haemodialysis and related therapies, http://www.iso.org/iso/ catalogue_detail.htm?csnumber=61863

17. Administration, Center for Devices and Radiological Health, Center for Biologics Evaluation and Research. General principles of software validation. Final Guidance for Industry and FDA Staff Document issued on: January 11 , 2002. Available at http://www.fda.gov/cdrh/comp/guidance/938.html.

18. Tsuchiya S, Moriishi M, Takahashi N, Watanabe H, Kawanishi H, Kim ST, et al. Experience with the JMS fully automated dialysis machine. ASAIO J. 2003:49:547-53.
19. Kawanishi H, Moriishi M, Sato T, Taoka M. Fully automated dialysis system based on the central dialysis fluid delivery system. Blood Purif. 2009;27 Suppl 1:56-63.

20. Kawanishi H, Moriishi M. Fully automated dialysis system for online hemodiafiltration built into the central dialysis fluid delivery system. Contrib Nephrol. 2011;168:107-16.

\section{Submit your next manuscript to BioMed Central and take full advantage of:}

- Convenient online submission

- Thorough peer review

- No space constraints or color figure charges

- Immediate publication on acceptance

- Inclusion in PubMed, CAS, Scopus and Google Scholar

- Research which is freely available for redistribution

Submit your manuscript at www.biomedcentral.com/submit 